\title{
ERK5 positively regulates cigarette smoke-induced urocystic epithelial-mesenchymal transition in SV-40 immortalized human urothelial cells
}

\author{
HAO GENG $^{1 *}$, LI ZHAO ${ }^{1 *}$, ZHAOFENG LIANG ${ }^{2}$, ZHIQIANG ZHANG $^{1}$, DONGDONG XIE $^{1}$, LIANGKUAN BI $^{1}$, \\ YI WANG ${ }^{1}$, TAO ZHANG ${ }^{1}$, LEI CHENG ${ }^{1}$, DEXIN YU ${ }^{1}$ and CAIYUN ZHONG ${ }^{2}$ \\ ${ }^{1}$ Department of Urology, The Second Affiliated Hospital of Anhui Medical University, Hefei, Anhui 230032; \\ ${ }^{2}$ Department of Toxicology, School of Public Health, Nanjing Medical University, Nanjing, Jiangsu 211166, P.R. China
}

Received March 19, 2015; Accepted June 15, 2015

DOI: $10.3892 /$ or.2015.4130

\begin{abstract}
Bladder cancer is universally acknowledged as a significant public health issue. Abundant evidence shows that cigarette smoke (CS) is the primary risk factor for bladder cancer. However, the mechanism of CS-induced bladder cancer has not been fully elucidated. CS-induced epithelialmesenchymal transition (EMT) is critically involved in cell malignant transformation. The role of ERK5, the lesser studied member of the MAPK family, in regulating CS-triggered EMT has not yet been investigated. The objective of the present study was to investigate the regulatory role of ERK5 in CS-induced urocystic EMT. SV-40 immortalized normal human urothelial cells (SV-HUC-1) were used as in vitro CS exposure models. EMT phenotypic alterations were assessed by changes in cell morphology, invasive capacity, as well as expression of epithelial and mesenchymal markers. Protein and mRNA expression levels were analyzed by western blotting and quantitative reverse transcriptase-polymerase chain reaction (qRT-PCR). ERK5 inhibition studies were performed with a specific inhibitor. Exposure of SV-HUC-1 cells to CS induced morphological change, enhanced invasive capacity, reduced epithelial marker expression and increased mesenchymal marker expression. Importantly, we demonstrated for the first time that ERK5 positively regulated CS-mediated
\end{abstract}

Correspondence to: Professor Dexin Yu, Department of Urology, The Second Affiliated Hospital of Anhui Medical University, 678 Furong Road, Hefei, Anhui 230032, P.R. China

E-mail: yudx_urology@126.com

Professor Caiyun Zhong, Department of Toxicology, School of Public Health, Nanjing Medical University, Nanjing, Jiangsu 211166, P.R. China

E-mail: cyzhong@njmu.edu.cn

*Contributed equally

Key words: bladder cancer, cigarette smoke, epithelial-mesenchymal transition, ERK5 pathway, AP-1
EMT in urothelial cells, as evidenced by the findings that CS promoted ERK5 activation, and that the CS-triggered alteration in the EMT phenotype was reversed by ERK5 inhibition.

\section{Introduction}

Bladder cancer is the fifth most common cancer in Western countries, responsible for more than 130,000 deaths worldwide annually, and is the first leading cause of death among urinary malignancies in China $(1,2)$. Cigarette smoking is estimated to cause $\sim 50 \%$ of the bladder cancer cases in males and $25 \%$ of the bladder cancers in females in the US. At any point in time, 2.7 million people have a history of bladder cancer (3).

Smoking, specific industrial chemicals, dietary nitrates and arsenic represent the most important exogenous risk factors for bladder cancer (4). Cigarette smoke (CS) is the main risk factor for bladder cancer $(4,5)$. In a meta-analysis of 43 published case-control and cohort studies, Zeegers et al concluded that current cigarette smokers have an $\sim 3$-fold higher risk of developing bladder cancer than non-smokers (6). There are more than 60 established carcinogens, which occur in mainstream smoke, side-stream smoke, and the particulate phase of cigarette smoking extract (CSE), such as polycyclic aromatic hydrocarbons (PAHs), benzo[a]pyrene (BaP), nitrosamines and aromatic amines (7). Although enormous progress has been made in exploring how CS leads to bladder cancer development, the molecular pathogenesis remains largely unknown.

Epithelial-mesenchymal transition (EMT) is a multistep process in which epithelial cells lose their epithelial characteristics and gain mesenchymal characteristics, such as motility and invasive properties (8). Numerous in vitro and in vivo studies suggest that EMT is associated with cancer cell invasion and metastasis in various malignancies, including bladder cancer. In addition to facilitating tumor invasion and metastasis, EMT is also critically involved in the initiation of tumorigenesis by promoting cell malignant transformation (9-12). It has been reported that changes related to EMT are observed in the very earliest stages of oral malignancy and become more severe as lesions progress (13). Nonetheless, the underlying mechanisms by which CS induces EMT are poorly understood. 
The mitogen-activated protein kinases (MAPKs) include four major subfamilies: extracellular regulated protein kinases 1 and 2 (ERK1/2), the Jun N-terminal kinases (JNKs), p38 and ERK5 $(14,15)$. ERK5, also termed big MAPK1 (BMK1), is the least studied member of the MAPK family. MAPKs are responsible for the phosphorylation and activation of jun and fos proteins. The MEK5/ERK5 pathway is implicated in important cellular processes, including gene expression, proliferation, apoptosis, angiogenesis, cell motility and differentiation (16-19). While some studies have suggested the functions of ERK5 in cancer oncogenesis, its role in EMT regulation has not been well explored.

To date, no studies have been carried out to examine the action of ERK5 in CS-induced urocystic EMT. The purpose of the present study was to investigate CS-induced EMT in normal human urothelial cells. Furthermore, we discussed the potential role of the ERK5 pathway in CS-induced EMT, which may contribute to a better understanding of carcinogenesis related to CS, including bladder cancer.

\section{Materials and methods}

Materials. An SV-40 immortalized human urothelial cell line (SV-HUC-1) was purchased from the Chinese Academy of Typical Culture Collection Cell Bank. F12K medium was purchased from Gibco (New York, NY, USA). Fetal bovine serum (FBS) was obtained from PAA Laboratories (Pasching, Austria). 3-(4,5-Dimethylthiazol-2-yl)-2,5diphenyltetrazolium bromide (MTT) was purchased from Sigma-Aldrich. Phosphorylated ERK5, phosphorylated c-jun (p-c-jun), phosphorylated c-fos (p-c-fos), snail, slug, E-cadherin, $\mathrm{N}$-cadherin and vimentin were obtained from Cell Signaling Technology (Beverly, MA, USA). The antibody for ZO-1 was obtained from Santa Cruz Biotechnology (Santa Cruz, CA, USA). The GAPDH antibody was obtained from Biogot Technology (Nanjing, China). Primers for E-cadherin, ZO-1, N-cadherin, vimentin and GAPDH were synthesized by Invitrogen (Carlsbad, CA, USA). XMD8-92 was purchased from Beyotime (Shanghai, China). Sources of other materials are noted accordingly in the study.

Cell culture and treatment. SV-HUC-1 cells were cultured under an atmosphere of $5 \% \mathrm{CO}_{2}$ at $37^{\circ} \mathrm{C}$ in $\mathrm{F} 12 \mathrm{~K}$ medium containing antibiotics $(100 \mathrm{U} / \mathrm{ml}$ penicillin and $100 \mu \mathrm{g} / \mathrm{ml}$ streptomycin). The cells were seeded in $10 \mathrm{~cm}^{2}$ flasks. The medium was changed every other day until cells reached $80-90 \%$ confluence, and then treated with various concentrations of CS extract or with XMD8-92 $(5 \mu \mathrm{M})$.

Preparation of CSE. CSE was freshly prepared for each experiment by combusting one commercial cigarette according to the reported method $(20,21)$. Commercial cigarettes (Hongtashan filter-tipped cigarettes made in Yunnan, China; each contain $12 \mathrm{mg}$ tar and $1.1 \mathrm{mg}$ nicotine) were smoked. By application of a vacuum, mainstream smoke was drawn through $10 \mathrm{ml}$ of prewarmed $\left(37^{\circ} \mathrm{C}\right) \mathrm{FBS}$-free $\mathrm{F} 12 \mathrm{~K}$ medium supplemented with penicillin and streptomycin at a rate of $5 \mathrm{~min} /$ cigarette. The obtained solution was referred to as having $100 \%$ strength. Then the CSE stock solution was filtered through a $0.22-\mu \mathrm{m}$ pore size filter and diluted to the desired concentration with treatment medium. The resulting CSE was applied to epithelial cell cultures within $30 \mathrm{~min}$ of preparation. The control solution was prepared using the same protocol, except that the cigarettes were unlit.

Cell toxicity assay. SV-HUC-1 cells were seeded in 96-well plates at a plating density of $2 \times 10^{3}$ cells/well in $200 \mu \mathrm{l}$ of medium. Then the cells were exposed to various concentrations of CSE prepared as previously outlined for 5 days, and the cell viability was determined by MTT assay. For the present study, media containing various concentrations of CSE were changed every day. Five days later, MTT stock solution was added to each well to solubilize the formazan crystals, and plates were incubated for an additional $4 \mathrm{~h}$ at $37^{\circ} \mathrm{C}$. Afterwards, MTT solution in the medium was removed and the crystals were solubilized in dimethylsulfoxide (DMSO). Absorbance was measured at $490 \mathrm{~nm}$ using a microplate reader. All measurements were performed in triplicate.

Transwell assay. The invasion assays were performed in a 24 -well Boyden chamber with an $8-\mu \mathrm{m}$ pore size polycarbonate membrane (Millipore, Billerica, MA, USA) coated with Matrigel to form a matrix barrier. A total of $100 \mu \mathrm{l}$ of serum-free medium (containing $1 \times 10^{4}$ cells) was added to the upper compartment of the chamber, whereas the lower compartment was filled with $800 \mu \mathrm{l}$ of F12K supplemented with $10 \%$ FBS. After incubation at $37^{\circ} \mathrm{C}$ for $48 \mathrm{~h}$, the SV-HUC-1 cells remaining inside the upper chamber were removed with cotton swabs. The cells on the lower surface of the membrane were stained with $0.1 \%$ crystal violet after fixation with methanol and then imaged under a light microscope. Subsequently, the cells were bleached with glacial acetic acid and the absorbance of the eluant was measured at $570 \mathrm{~nm}$.

Western blot analysis. SV-HUC-1 cells were harvested, washed with ice-cold phosphate-buffered saline (PBS) and lysed in RIPA buffer (Thermo Scientific, USA). Concentrations of the precipitated proteins in the cell lysates were measured with BCA protein assay (Pierce, Rockford, IL, USA). Afterwards, the proteins were diluted to equal concentrations, boiled for 5 min and separated by 7.5-10\% SDS-PAGE, transferred onto polyvinylidene difluoride membranes (Millipore). After blocking with $5 \%$ milk, the membranes were blocked and incubated with the indicated primary antibodies and secondary antibodies. The blots were subsequently developed using an enhanced chemiluminescence detection kit (Amersham Biosciences, USA) and exposed to film. GAPDH served as the loading control.

Quantitative reverse transcription-polymerase chain reaction ( $q R T-P C R)$. Total RNA was isolated by RNAiso Plus according to the manufacturer's instructions. (Takara, Japan). Then total RNA was transcribed into cDNA using AMV reverse transcriptase (Takara) following the manufacturer's protocol. qRT-PCR was performed using the Power SYBR-Green Master Mix (Takara) and an ABI 7300 Real-Time PCR Detection System (Applied Biosystems). All of the primers were synthesized by Invitrogen. Respectively, normalization was achieved by dividing the expression level of mRNA by its respective GAPDH expression level. Fold-change in the 


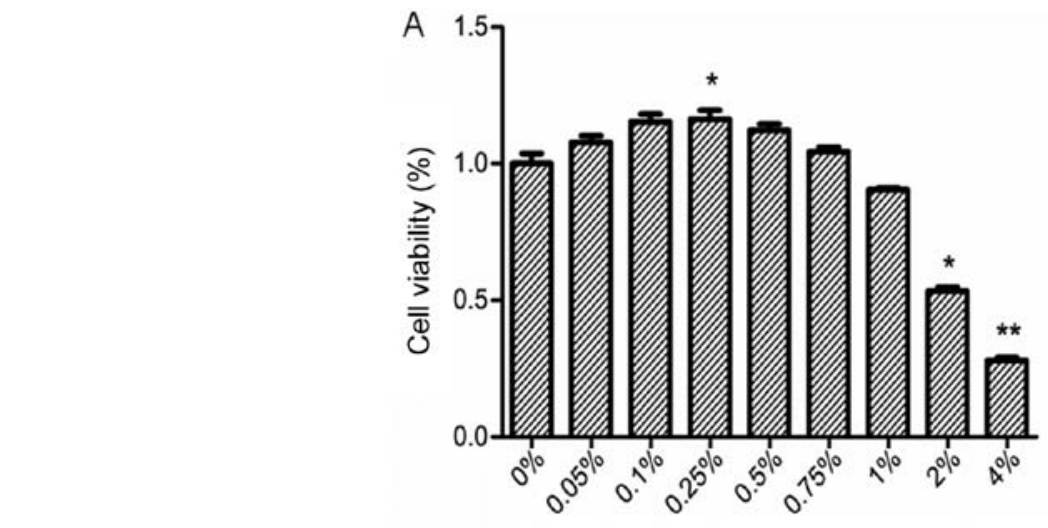

B

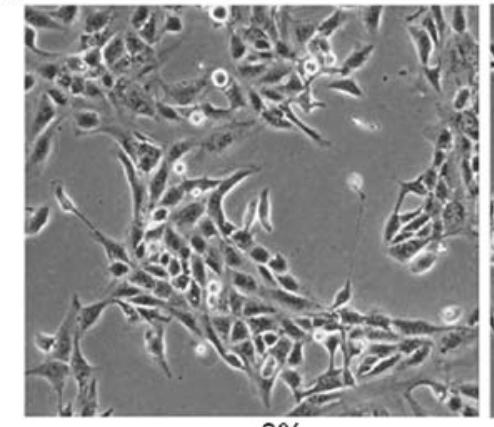

$0 \%$

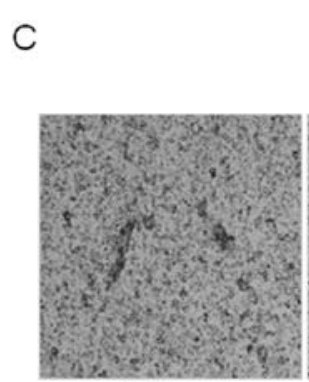

$0 \%$

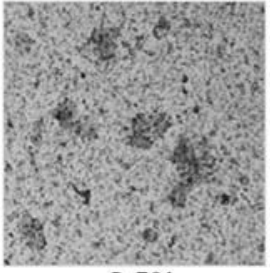

$0.5 \%$

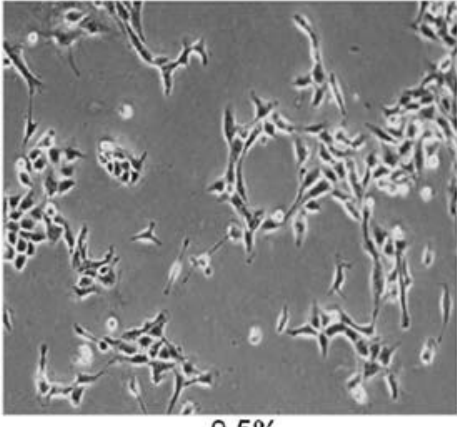

$0.5 \%$
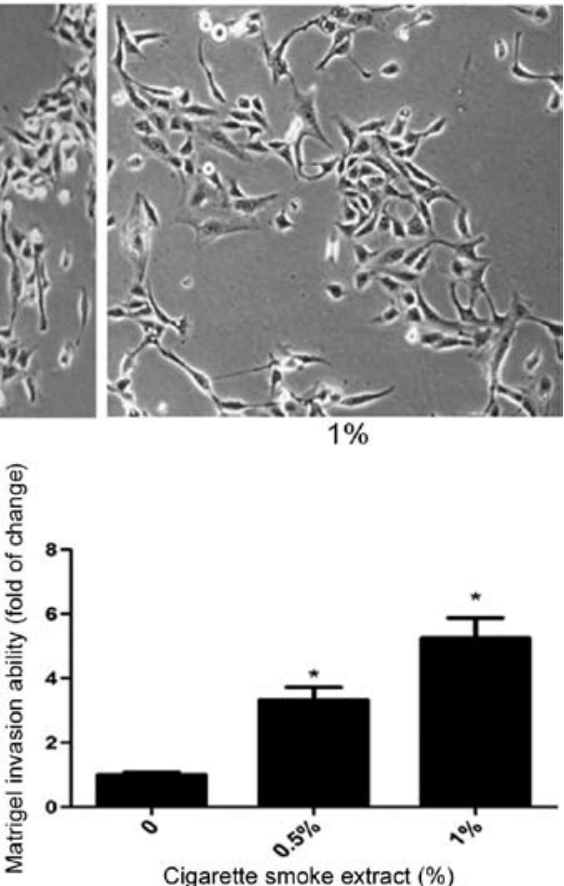

Figure 1. CS induces EMT morphological change and enhances the invasive capacity of normal human urothelial (SV-HUC-1) cells. (A) Effect of cigarette smoke extract (CSE) on the cell viability of SV-HUC-1 cells. The results showed that lower concentrations of CSE (0.05-0.5\%) induced mildly enhanced cell viability in the SV-HUC-1 cells. Additionally, cell viability decreased below $80 \%$ when cells were exposed to $2 \%$ or higher CSE concentrations, which proved to be toxic to the SV-HUC-1 cells. (B) CSE induced morphological change from an epithelial to a spindle-like mesenchymal shape, as shown by morphological examination of the SV-HUC-1 cells following CSE treatment for 5 days. SV-HUC-1 cells became longer and thinner, some of which generated a slender tail. (C) CSE enhanced the invasive capacity of the SV-HUC-1 cells, as determined by Transwell invasion assay. The $0.5 \%$ CSE and $1 \%$ CSE groups revealed that CSE had a strong stimulative effect on the invasion capacity of the SV-HUC-1 cells. The subsequent absorbance assay confirmed this change. Data are expressed as mean $\pm \mathrm{SD}$. ${ }^{*} \mathrm{P}<0.05,{ }^{* *} \mathrm{P}<0.01$, compared with the control group. CS, cigarette smoke; EMT, epithelial-mesenchymal transition.

expression of each gene was calculated by a comparative threshold cycle $(\mathrm{Ct})$ method using the formula $2^{-\Delta \Delta \mathrm{Ct}}$. The primers used were: E-cadherin forward, 5'-TCGACACCCGA TTCAAAGTGG-3' and reverse, 5'-TTCCAGAAACGGAGGC CTGAT-3'; vimentin forward, 5'-CCTTGACATTGAGATT GCCA-3' and reverse, 5'-GTATCAACCAGAGGGAGTGA-3'; ZO-1 forward, 5'-GCAGCCACAACCAATTCATAG-3' and reverse, 5'-GCAGACGATGTTCATAGTTTC-3'; N-cadherin forward, 5'-ATCAAGTGCCATTAGCCAAG-3' and reverse, 5'-CTGAGCAGTGAATGTTGTCA-3'; GAPDH forward, 5'-GCTGCCCAACGCACCGAATA-3' and reverse, 5'-GAGT CAACGGATTTGGTCGT-3'.

Statistical analysis. Statistical analyses were performed with SPSS 16.0. All data are expressed as mean \pm standard deviation. One-way ANOVA was used for comparison of statistical differences among multiple groups, followed by the LSD significant difference test. In case of comparison between two groups, an unpaired Student's t-test was used. A value of $\mathrm{P}<0.05$ was considered to indicate a statistically significant difference.

\section{Results}

CS induces EMT in SV-HUC-1 cells. CS is the most important risk factor for bladder cancer, and CS-induced EMT is critically involved in CS-associated malignant transformation. To study the cell viability of the SV-HUC-1 cells after treatment with CSE, the cells were incubated with CSE $(0$, $0.05,0.1,0.25,0.5,0.75,1,2$ and $4 \%$ ) for 5 days and examined by MTT assay. The results showed that the cell viability decreased below $80 \%$ when the cells were exposed to $2 \%$ or higher CSE concentrations, which proved to be toxic to the 


\section{A E-cadherin}

Zo-1

Vimentin

$\mathrm{N}$-cadherin

GAPDH
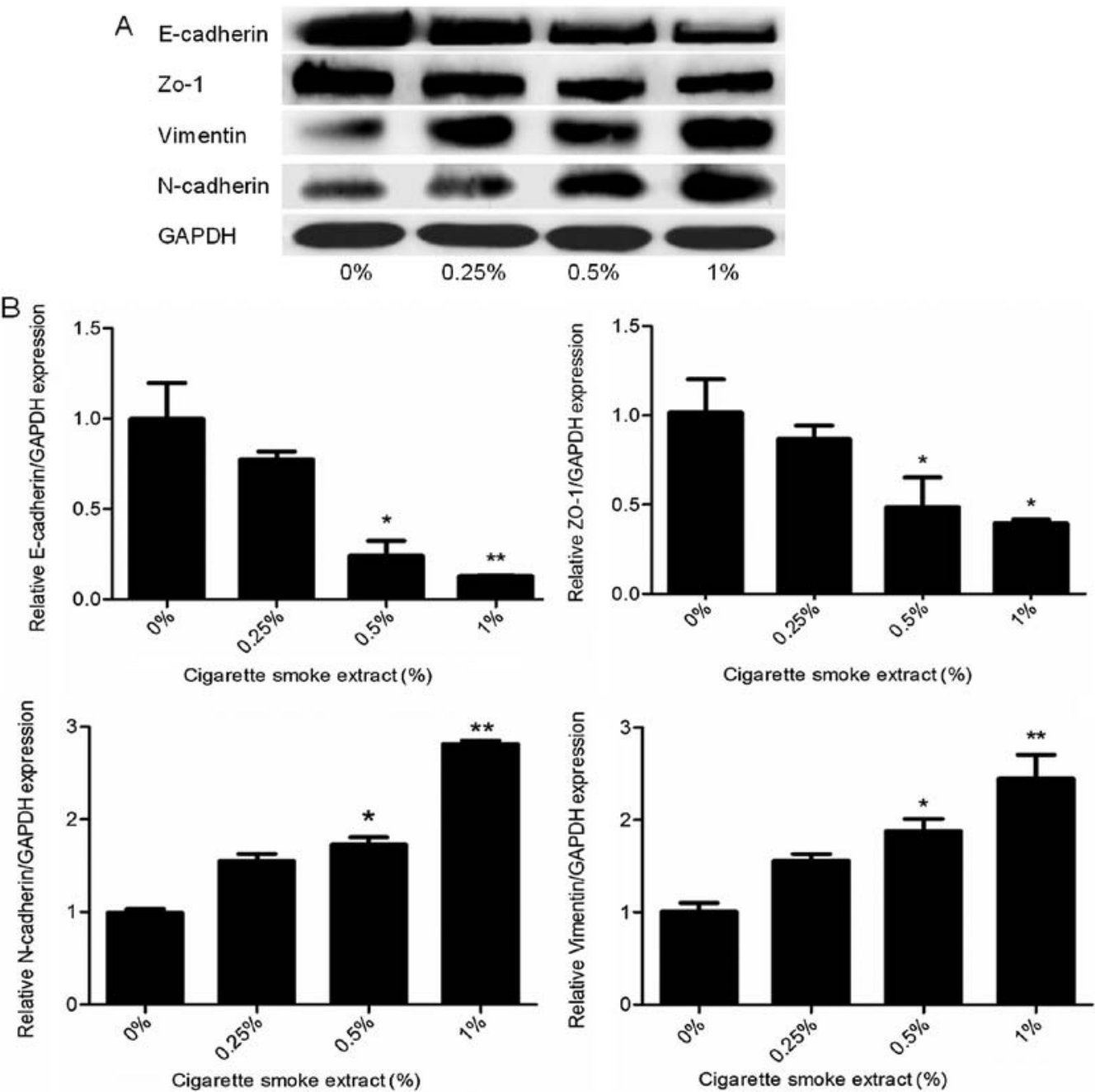

Figure 2. CS alters the expression of EMT markers in SV-HUC-1 cells following CSE treatment for 5 days. (A) Cigarette smoke extract (CSE) increased the protein expression of epithelial markers E-cadherin and ZO-1, and decreased protein expression of mesenchymal markers vimentin and $\mathrm{N}$-cadherin in the SV-HUC-1 cells, as shown by western blotting. GAPDH was used as a loading control. (B) CSE enhanced the expression of E-cadherin and ZO-1 mRNAs, and decreased the expression of vimentin and N-cadherin mRNAs, as detected by quantitative reverse transcriptase-polymerase chain reaction after normalization to GAPDH. Data are expressed as mean $\pm \mathrm{SD}$. ${ }^{*} \mathrm{P}<0.05,{ }^{* *} \mathrm{P}<0.01$, compared with the control group. CS, cigarette smoke; EMT, epithelial-mesenchymal transition.

SV-HUC-1 cells (Fig. 1A). Therefore, 1\% CSE was selected as the maximum concentration for the following experiments.

The process of EMT is manifested by alterations in cell morphology and invasive capacity, as well as expression of epithelial and mesenchymal markers. Treatment of the SV-HUC-1 cells with CSE for 5 days resulted in significant morphological change from a urothelial oblate-shape to a spindle-like mesenchymal form (Fig. 1B). Additionally, the cells became dispersive and some SV-HUC-1 cells even generated a tail-like change after treatment with CSE. To further examine the effect of CSE on EMT, Transwell assays were carried out to analyze SV-HUC-1 cell invasive capacities in response to $\mathrm{CSE}$. Invasion of the cells through reconstituted Matrigel matrices was enhanced by CSE (Fig. 1C). To determine whether molecular alterations of EMT occurred in the CSE-treated cells, the expression levels of EMT markers were determined. Exposure of the SV-HUC-1 cells to CSE resulted in decreased protein expression of the epithelial markers E-cadherin and ZO-1. In contrast, the protein levels of mesenchymal markers vimentin and $\mathrm{N}$-cadherin were increased, as

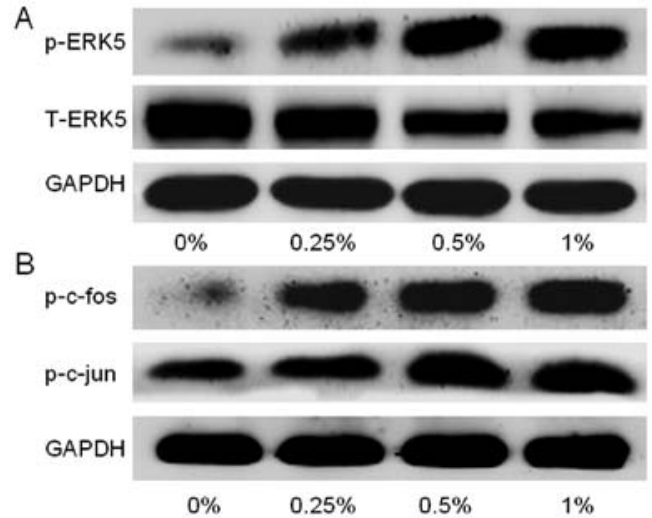

Figure 3. CS-induced EMT is associated with activation of ERK5 in SV-HUC-1 cells. (A) CSE activated ERK5 activation in the SV-HUC-1 cells following CSE treatment for 5 days. The levels of phosphorylated ERK5 (p-ERK5), the active form of ERK5, as well as total ERK5 (T-ERK5), were measured by western blotting following CSE treatment. (B) CSE increased AP-1 protein activation following CSE treatment for 5 days, as shown by elevated levels of phosphorylated c-Fos (p-c-fos) and phosphorylated c-Jun (p-c-jun). GAPDH was used as a loading control. CS, cigarette smoke; EMT, epithelial-mesenchymal transition. 

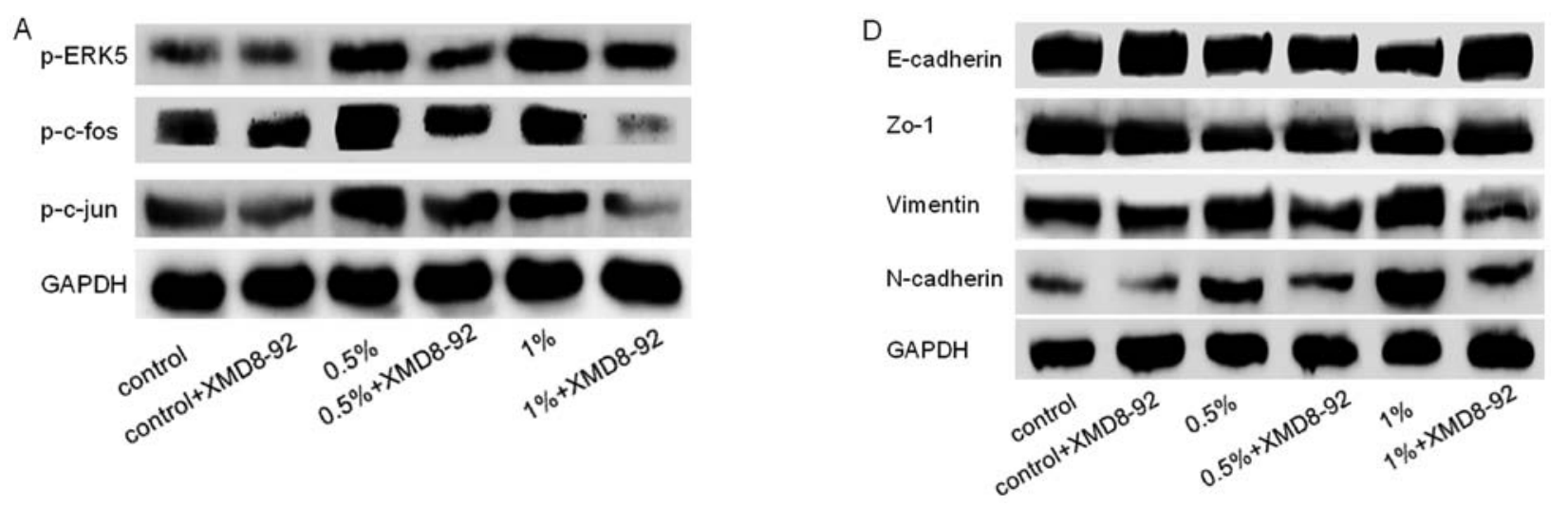

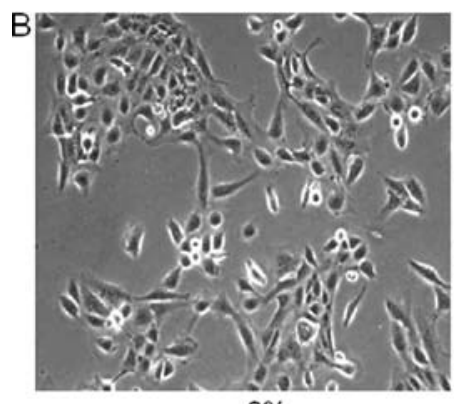

$0 \%$

C

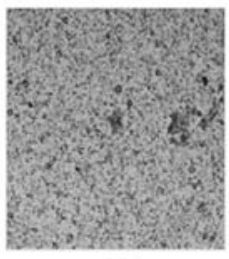

$0 \%$

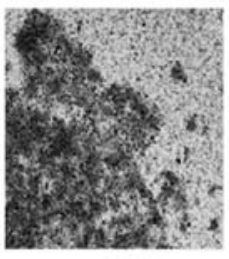

$0.5 \%$

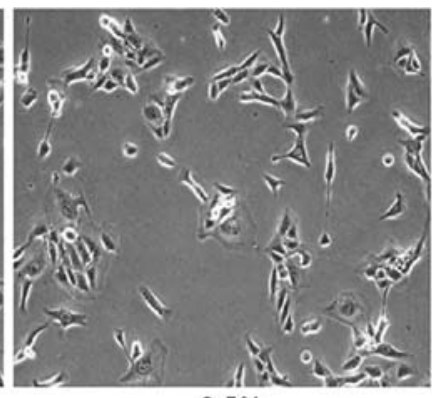

$0.5 \%$

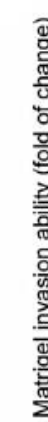

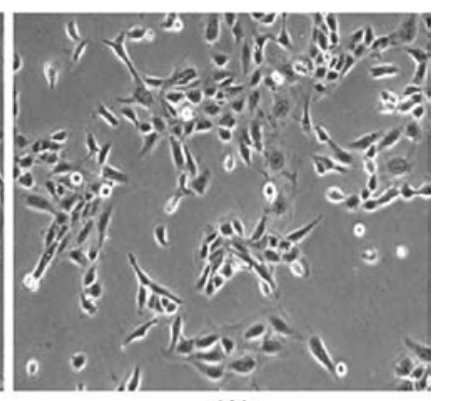

$1 \%$

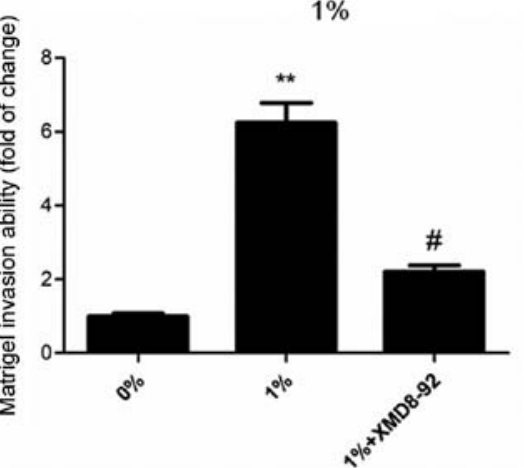

Figure 4. Inhibition of ERK5 attenuates CS-induced EMT in SV-HUC-1 cells. (A) ERK5 inhibitor XMD8-92 suppressed ERK5 activation in the SV-HUC-1 cells. SV-HUC-1 cells were treated with various concentrations of CSE together with a highly specific ERK5 inhibitor (XMD8-92) for 5 days, and western blot analyses were performed for the measurements of p-ERK5, p-c-Fos and p-c-Jun. The control group represented 0\% CSE + DMSO. XMD8-92 suppressed the activation of p-ERK5, p-c-Fos and p-c-Jun induced by CS exposure. Moreover, XMD8-92 had little effect on the control group. (B) XMD8-92 attenuated the morphological change of SV-HUC-1 cells. Compared with the CSE group, the SV-HUC-1 cells became collective and full after treatement with XMD8-92. (C) XMD8-92 decreased the invasive capacity of the SV-HUC-1 cells, as determined by the invasion assay. Results showed that the enhanced invasion capacity of the SV-HUC-1 cells triggered by 1\% CSE was reversed by XMD8-92. (D) Treatment of ERK5 inhibitor XMD8-92 along with 0.5\% CSE or 1\% CSE resulted in enhanced protein levels of E-cadherin and ZO-1, and decreased protein levels of vimentin and N-cadherin.

shown by western blot analyses (Fig. 2A). Moreover, qRT-PCR analyses revealed similar changes in the mRNA levels of epithelial and mesenchymal markers in the SV-HUC-1 cells exposed to CSE (Fig. 2B). Moreover, the higher the concentration of CSE, the more obvious was the observed change. Collectively, data from the morphological, invasive and molecular changes demonstrated that CS exposure induced EMT in human urothelial cells in a dose-dependent manner.

CS-induced urocystic EMT is associated with ERK5 activation. As the lesser studied member of the MAPK family, ERK5 is implicated in cancer oncogenesis. The function of ERK5 in EMT regulation has not been well explored, and several lines of evidence suggest that a differential regulatory role of ERK5 in EMT exists. To determine whether CS-elicited urothelial EMT is associated with a change in ERK5 activation, the level of phosphorylated ERK5, an indicator of ERK5 activation was measured. Exposure of the SV-HUC-1 cells to CSE for 5 days dose-dependently activated the level of phosphorylated ERK5 (p-ERK5) and simultaneously restrained the level of total ERK5, suggesting a stimulative effect of CS on ERK5 activity (Fig. 3A). Meanwhile, CS increased the activation of AP-1 protein in the SV-HUC-1 cells, as indicated by elevated levels of p-c-jun and p-c-fos (Fig. 3B).

Inhibition of ERK5 attenuates CS-induced EMT in SV-HUC-1 cells. Since the above results revealed that CS-induced EMT was associated with ERK5 activation in SV-HUC-1 cells, we next explored the role of ERK5 in urocystic EMT regulation. SV-HUC-1 cells were treated for 5 days with $5 \mu \mathrm{M}$ XMD8-92, a highly specific ERK5 inhibitor which suppresses ERK5 autophosphorylation and does not inhibit ERK1/2 activity $(22,23)$. 

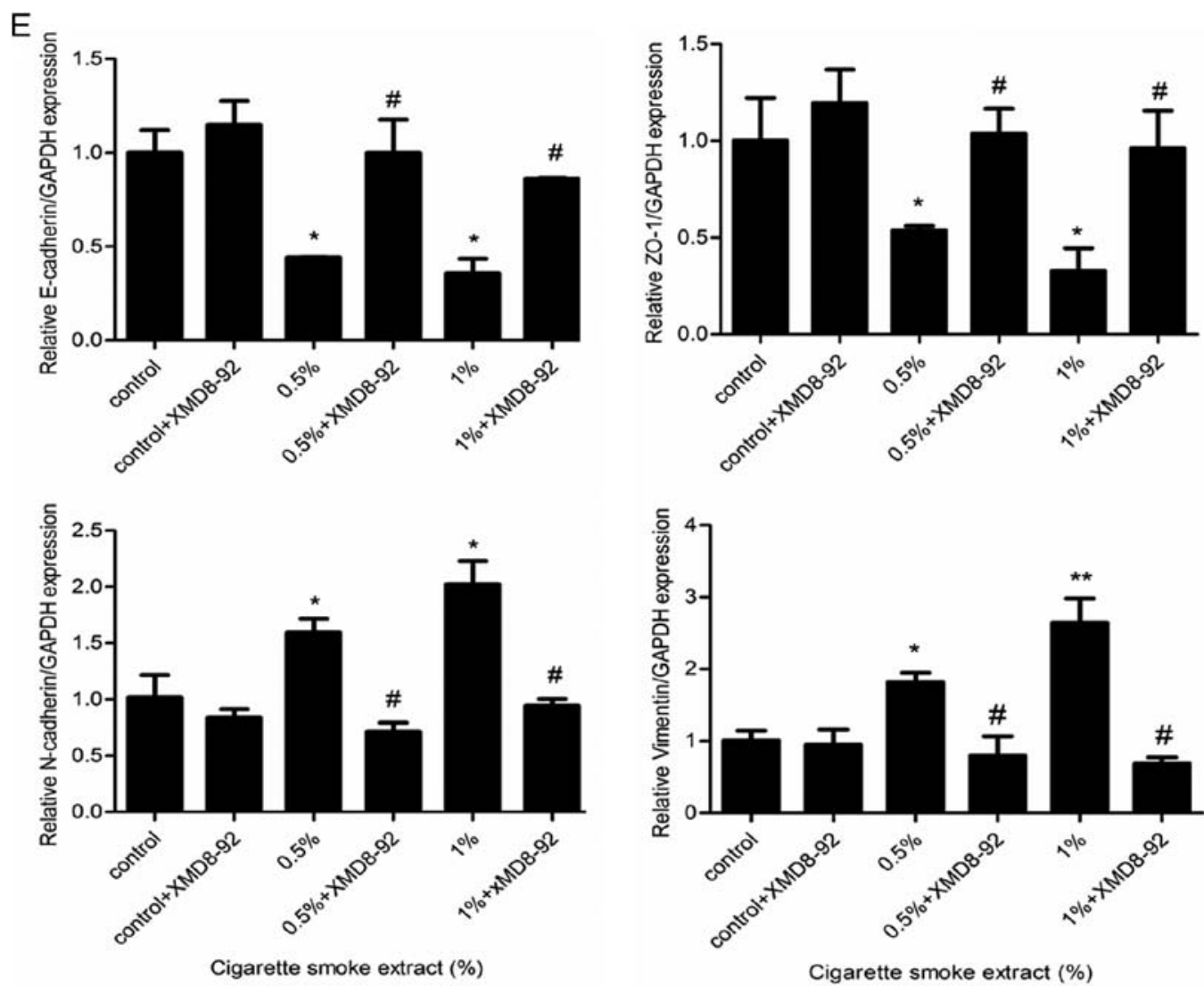

Figure 4. Continued. (E) Treatment with the ERK5 inhibitor XMD8-92 along with 0.5\% CSE or 1\% CSE increased the expression of E-cadherin and ZO-1 mRNAs, and decreased vimentin and $\mathrm{N}$-cadherin mRNAs. All the data are expressed as mean $\pm \mathrm{SD}$. ${ }^{*} \mathrm{P}<0.05$ and ${ }^{* *} \mathrm{P}<0.01$, CSE group compared with the control group. " $\mathrm{p}<0.05$, XMD8-92 group compared with the srespective CSE group. CS, cigarette smoke; EMT, epithelial-mesenchymal transition; DMSO, dimethylsulfoxide.

As expected, XMD8-92 downregulated p-ERK5 and levels of p-c-jun and p-c-fos in the SV-HUC-1 cells (Fig. 4A). XMD8-92 treatment reversed the mensenchymal-like morphological change in the cells (Fig. 4B). Moreover, XMD8-92 also restrained the invasive capacity of the SV-HUC-1 cells, as determined by Transwell assays (Fig. 4C). Furthermore, inhibition of ERK5 by XMD8-92 resulted in upregulation of the protein and mRNA levels of the epithelial markers E-cadherin and ZO-1, as well as downregulation of the mesenchymal markers vimentin and N-cadherin (Fig. 4D and E). Therefore, these results suggest that ERK5 activity plays an important role in CSE-induced EMT in SV-HUC-1 cells.

\section{Discussion}

Bladder cancer is one of the leading causes of cancer-related death worldwide. The relationship between the occurrence of urocystic cancer and cigarette smoke (CS) has been established $(24,25)$. CS is the primary cause of bladder cancer, which promotes the initiation and progression of bladder tumorigenesis. The underlying molecular mechanisms by which CS causes bladder cancer development remain to be established. In the present study, we revealed that CS induced EMT in SV-HUC-1 cells. Most importantly, we demonstrated for the first time that ERK5 positively regulated CS-mediated EMT, as evidenced by the findings that CS promoted ERK5 activation and that CS-triggered EMT phenotypic alteration was reversed by ERK5 inhibition. These findings suggest the important role of ERK5 activity in CS-associated EMT and provide critical information concerning the molecular mechanisms of CS-related bladder tumorigenesis as well as the search for potential targets of bladder cancer intervention.

Characterized by changes in cell morphology, migration and invasion capacity, as well as the expression profile of epithelial and mesenchymal markers, EMT is a crucial process in cancer development. Evidence has revealed that exposure of cells to carcinogens induces EMT during transformation and tumor formation $(10,11,26)$, suggesting the important role of EMT in the initiation of tumorigenesis by promoting cell malignant transformation. Likewise, Cavallaro et al put forward the cadherin switch theory. That is, epithelial cells undergo mesenchymal cell phenotype conversion, accompanied by loss of E-cadherin and gain of $\mathrm{N}$-cadherin, which strongly supports the view that malignant tumors develop from benign tumors (27). E-cadherin plays an essential role in epithelial cell-to-cell interactions since it mediates the connections between adjacent epithelial cells and maintains the phenotype and apical-base polarity of epithelial cells (28). It has been documented that CS promotes EMT, resulting in loss of cellular polarity, downregulation of epithelial cadherin, loss of cell-cell adhesion and increased mobility of epithelial cells (29). In agreement with previous studies, we showed in the present study that exposure to CS induced EMT in SV-HUC-1 cells, as manifested by morphological change from an epithelial to a mesenchymal 
form, increased invasive capacity, as well as alterations in the expression of EMT markers, including decreased E-cadherin and increased $\mathrm{N}$-cadherin, which powerfully verify the cadherin switch theory. Taken together, our data proved that CS triggers EMT in vitro settings.

To date, no studies have been carried out to investigate the function of ERK5 in CS-induced EMT or in bladder cancer cell invasion and metastasis. In the present study, we showed that CS-induced EMT was associated with activation of ERK5 in vitro. To determine the role of ERK 5 in urocystic EMT regulation, the inhibitory effect of ERK5 on CS was mimicked with a highly specific ERK5 inhibitor. Inhibition of ERK5 reversed the mesenchymal-like morphological changes triggered by CS. Moreover, the enhanced invasive capacities as well as alterations in EMT marker expression were attenuated by inhibition of ERK5. Collectively, these data clearly indicated that ERK5 positively regulated CS-induced urocystic EMT.

In regards to proto-oncogenic signaling, the MAPK pathways are generally thought to promote cancer development; while differential biological functions exist for individual MAPK pathways. Among the MAPK family, ERK5 is twice the size of the other members. The N-terminal half of ERK5 contains the kinase domain which is similar to that of ERK1/2 and has the TEY activation motif. Unlike other MAPKs, the C-terminal region of ERK5 contains a long non-catalytic domain which has a unique function. Upon activation, ERK5 phosphorylates and activates downstream target molecules, including transcription factors such as members of the AP-1 proteins (30). Activation of ERK5 also results in autophosphorylation of the C-terminus, which alone has the ability to increase transcriptional activity $(31,32)$. Therefore, ERK5 differs from other MAPKs in possessing transcriptional activation activity. It is able to regulate the transcription of downstream molecules in two ways, i.e., through either the phosphorylation or the enhancement of the transcription activity of target molecules (16-19). Nevertheless, only a small number of molecules have been identified as target genes of ERK5. Notably, it has been found that ERK5 transcriptionally regulates gene expression in a tissue-specific manner (33). In skeletal muscle cells, activation of ERK5 significantly inhibits TNF- $\alpha$-mediated NF- $\kappa$ B activity (34). In lung microvascular endothelial cells, activation of ERK5 suppresses HIF1 $\alpha$ expression and inhibits angiogenesis (35). In the present study, we showed that CS-mediated ERK5 activation induced the activation of AP-1 proteins c-Fos and c-Jun. Correspondingly, inhibition of ERK5 decreased c-Fos and c-Jun activation, suggesting the positive modulation of ERK5 on the AP-1 pathway. It is noteworthy that the precise mechanism by which ERK5 regulates AP-1 activation is unknown at the present time, and future studies are warranted to define the underlying mechanism of urocystic ERK5 regulation on AP-1 protein.

As previously mentioned, XMD8-92 is a highly specific inhibitor of ERK5. XMD8-92 can be competitively combined with ATP which effectively inhibits ERK5 autophosphorylation and does not affect the activity of ERK1/2. In animal experiments, treating lung xenograft tumors of mouse with XMD8-92, effectively inhibits tumor growth. This inhibition is accomplished through inhibition of ERK5 phosphorylation and inhibition of the PML gene (35). Notably, research has also shown that XMD8-92 significantly inhibits the activity of CDKI, thus as to promote the proliferation of human breast cancer cells (36). At present, there is no study on the application of XMD8-92 in bladder urothelial cells. Thus, the present study is the first to confirm that ERK5 positively regulates EMT in urothelial cells triggered by CS. Based on the important role of the ERK5 signaling pathway in the development of cancer, a new choice has been provided for tumor treatment. Nevertheless, how to block the ERK5 signaling pathway in a reasonable manner requires further exploration.

In summary, exposure to CS induced EMT in normal urothelial cells and activated the ERK5 pathway and the transcription factor AP-1, while the ERK5 inhibitor inhibited AP-1 activity and afterwards restrained the EMT triggered by CS. The present study effectively elucidated the underlying molecular mechanisms involved during the occurrence of bladder tumors related to CS. Furthermore, inhibition of the EKR5 pathway may be a therapeutic target for the treatment of early stage bladder cancer.

\section{Acknowledgements}

The present study was supported by grants from the National Natural Science Foundation of China (nos. 81373005, 81072330 and 81202194), and by the Priority Academic Program Development of Jiangsu Higher Education Institutions (PAPD).

\section{References}

1. Jemal A, Bray F, Center MM, Ferlay J, Ward E and Forman D: Global cancer statistics. CA Cancer J Clin 61: 69-90, 2011.

2. Siegel R, Naishadham D and Jemal A: Cancer statistics, 2012. CA Cancer J Clin 62: 10-29, 2012.

3. Ploeg M, Aben KK and Kiemeney LA: The present and future burden of urinary bladder cancer in the world. World J Urol 27: 289-293, 2009.

4. Volanis D, Kadiyska T, Galanis A, Delakas D, Logotheti S and Zoumpourlis V: Environmental factors and genetic susceptibility promote urinary bladder cancer. Toxicol Lett 193: 131-137, 2010.

5. Boffetta P: Tobacco smoking and risk of bladder cancer. Scand J Urol Nephrol Suppl 42 (s218): S45-S54, 2008.

6. Zeegers MP, Tan FE, Dorant E and van Den Brandt PA: The impact of characteristics of cigarette smoking on urinary tract cancer risk: A meta-analysis of epidemiologic studies. Cancer 89: 630-639, 2000.

7. Hecht SS: Tobacco carcinogens, their biomarkers and tobaccoinduced cancer. Nat Rev Cancer 3: 733-744, 2003.

8. Kalluri R and Weinberg RA: The basics of epithelial-mesenchymal transition. J Clin Invest 119: 1420-1428, 2009.

9. Sun JL, Chen DL, Hu ZQ, Xu YZ, Fang HS, Wang XY, Kan L and Wang SY: Arsenite promotes intestinal tumor cell proliferation and invasion by stimulating epithelial-to-mesenchymal transition. Cancer Biol Ther 15: 1312-1319, 2014.

10. Xu W, Ji J, Xu Y, Liu Y, Shi L, Liu Y, Lu X, Zhao Y, Luo F, Wang B, et al: MicroRNA-191, by promoting the EMT and increasing CSC-like properties, is involved in neoplastic and metastatic properties of transformed human bronchial epithelial cells. Mol Carcinog 54 (Suppl 1): E148-E161, 2015.

11. Tellez CS, Juri DE, Do K, Bernauer AM, Thomas CL, Damiani LA, Tessema M, Leng S and Belinsky SA: EMT and stem cell-like properties associated with miR-205 and miR-200 epigenetic silencing are early manifestations during carcinogeninduced transformation of human lung epithelial cells. Cancer Res 71: 3087-3097, 2011.

12. Liu Y, Luo F, Xu Y, Wang B, Zhao Y, Xu W, Shi L, Lu X and Liu Q: Epithelial-mesenchymal transition and cancer stem cells, mediated by a long non-coding RNA, HOTAIR, are involved in cell malignant transformation induced by cigarette smoke extract. Toxicol Appl Pharmacol 282: 9-19, 2015.

13. Danielsson K, Wahlin YB, Coates PJ and Nylander K: Increased expression of Smad proteins, and in particular Smad3, in oral lichen planus compared to normal oral mucosa. J Oral Pathol Med 39: 639-644, 2010. 
14. Chang L and Karin M: Mammalian MAP kinase signalling cascades. Nature 410: 37-40, 2001.

15. Sangrar W, Shi C, Mullins G, LeBrun D, Ingalls B and Greer PA: Amplified Ras-MAPK signal states correlate with accelerated EGFR internalization, cytostasis and delayed HER 2 tumor onset in Fer-deficient model systems. Oncogene 27: 340, 2014.

16. Drew BA, Burow ME and Beckman BS: MEK5/ERK5 pathway: The first fifteen years. Biochim Biophys Acta 1825: 37-48, 2012

17. Nishimoto $\mathrm{S}$ and Nishida E: MAPK signalling: ERK5 versus ERK1/2. EMBO Rep 7: 782-786, 2006.

18. Hayashi M, Fearns C, Eliceiri B, Yang Y and Lee JD: Big mitogen-activated protein kinase 1/extracellular signal-regulated kinase 5 signaling pathway is essential for tumor-associated angiogenesis. Cancer Res 65: 7699-7706, 2005.

19. Wang $X$ and Tournier C: Regulation of cellular functions by the ERK5 signalling pathway. Cell Signal 18: 753-760, 2006.

20. Gál K, Cseh A, Szalay B, Rusai K, Vannay A, Lukácsovits J, Heemann U, Szabó AJ, Losonczy G, Tamási L, et al: Effect of cigarette smoke and dexamethasone on Hsp72 system of alveolar epithelial cells. Cell Stress Chaperones 16: 369-378, 2011.

21. Tian D, Zhu M, Chen WS, Li JS, Wu RL and Wang X: Role of glycogen synthase kinase 3 in squamous differentiation induced by cigarette smoke in porcine tracheobronchial epithelial cells. Food Chem Toxicol 44: 1590-1596, 2006.

22. Yang Q, Deng X, Lu B, Cameron M, Fearns C, Patricelli MP, Yates JR III, Gray NS and Lee JD: Pharmacological inhibition of BMK1 suppresses tumor growth through promyelocytic leukemia protein. Cancer Cell 14: 258-267, 2010.

23. Wang X, Pesakhov S, Weng A, Kafka M, Gocek E, Nguyen M Harrison JS, Danilenko M and Studzinski GP. ERK 5/MAPK pathway has a major role in $1 \alpha, 25-(\mathrm{OH})_{2}$ vitamin $\mathrm{D}_{3}$-induced terminal differentiation of myeloid leukemia cells. J Steroid Biochem Mol Biol 144 Pt A: 223-227, 2014.

24. Freedman ND, Silverman DT, Hollenbeck AR, Schatzkin A and Abnet CC: Association between smoking and risk of bladder cancer among men and women. JAMA 306: 737-745, 2011.

25. Alguacil J, Kogevinas M, Silverman DT, Malats N, Real FX, García-Closas M, Tardón A, Rivas M, Torà M, García-Closas R, et al: Urinary $\mathrm{pH}$, cigarette smoking and bladder cancer risk. Carcinogenesis 32: 843-847, 2011.

26. Zhao Y, Xu Y, Li Y, Xu W, Luo F, Wang B, Pang Y, Xiang Q, Zhou J, Wang X, et al: NF-kB-mediated inflammation leading to EMT via miR-200c is involved in cell transformation induced by cigarette smoke extract. Toxicol Sci 135: 265-276, 2013.
27. Cavallaro U, Schaffhauser B and Christofori G: Cadherins and the tumour progression: Is it all in a switch? Cancer Lett 176: $123-128,2002$.

28. Rangel MC, Karasawa H, Castro NP, Nagaoka T, Salomon DS and Bianco C: Role of Cripto-1 during epithelial-to-mesenchymal transition in development and cancer. Am J Pathol 180: 2188-2200, 2012.

29. Veljkovic E, Jiricny J, Menigatti M, Rehrauer H and Han W: Chronic exposure to cigarette smoke condensate in vitro induces epithelial to mesenchymal transition-like changes in human bronchial epithelial cells, BEAS-2B. Toxicol In Vitro 25: 446-453, 2011.

30. Kasler HG, Victoria J, Duramad O and Winoto A: ERK5 is a novel type of mitogen-activated protein kinase containing a transcriptional activation domain. Mol Cell Biol 20: 8382-8389, 2000.

31. Nagathihalli NS, Massion PP, Gonzalez AL, Lu P and Datta PK: Smoking induces epithelial-to-mesenchymal transition in non-small cell lung cancer through HDAC-mediated downregulation of E-cadherin. Mol Cancer Ther 11: 2362-2372, 2012.

32. Nithianandarajah-Jones GN, Wilm B, Goldring CE, Müller J and Cross MJ: ERK5: Structure, regulation and function. Cell Signal 24: 2187-2196, 2012.

33. Sohn SJ, Li D, Lee LK and Winoto A: Transcriptional regulation of tissue-specific genes by the ERK5 mitogen-activated protein kinase. Mol Cell Biol 25: 8553-8566, 2005.

34. Woo CH, Massett MP, Shishido T, Itoh S, Ding B, McClain C, Che W, Vulapalli SR, Yan C and Abe J: ERK5 activation inhibits inflammatory responses via peroxisome proliferator-activated receptor delta (PPARdelta) stimulation. J Biol Chem 281: 32164-32174, 2006.

35. Pi X, Garin G, Xie L, Zheng Q, Wei H, Abe J, Yan C and Berk BC: BMK1/ERK5 is a novel regulator of angiogenesis by destabilizing hypoxia inducible factor 1alpha. Circ Res 96: $1145-1151,2005$.

36. Perez-Madrigal D, Finegan KG, Paramo B and Tournier C: The extracellular-regulated protein kinase 5 (ERK5) promotes cell proliferation through the down-regulation of inhibitors of cyclin dependent protein kinases (CDKs). Cell Signal 24: 2360-2368, 2012. 